\title{
Bose-Einstein Quantum Phase Transition in an Optical Lattice Model
}

\author{
Michael Aizenman, Elliott H. Lieb, and Robert Seiringer \\ Department of Physics, Jadwin Hall, Princeton University, P.O. Box 708, Princeton NJ 08544, USA \\ Jan Philip Solove $*$ \\ School of Mathematics, Institute for Advanced Study, 1 Einstein Drive, Princeton NJ 08540, USA \\ Jakob Yngvason \\ Institut für Theoretische Physik, Universität Wien, Boltzmanngasse 5, A-1090 Vienna, Austria and \\ Erwin Schrödinger Institute for Mathematical Physics, Boltzmanngasse 9, A-1090 Vienna, Austria
}

(Dated: March 8, 2004)

\begin{abstract}
Bose-Einstein condensation (BEC) in cold gases can be turned on and off by an external potential, such as that presented by an optical lattice. We present a model of this phenomenon which we are able to analyze rigorously. The system is a hard core lattice gas at half-filling and the optical lattice is modeled by a periodic potential of strength $\lambda$. For small $\lambda$ and temperature, BEC is proved to occur, while at large $\lambda$ or temperature there is no BEC. At large $\lambda$ the low-temperature states are in a Mott insulator phase with a characteristic gap that is absent in the BEC phase. The interparticle interaction is essential for this transition, which occurs even in the ground state. Surprisingly, the condensation is always into the $\mathbf{p}=\mathbf{0}$ mode in this model, although the density itself has the periodicity of the imposed potential.
\end{abstract}

\section{INTRODUCTION}

One of the most remarkable recent developments in the study of ultracold Bose gases is the observation of a reversible transition from a Bose-Einstein condensate to a state composed of localized atoms as the strength of a periodic, optical trapping potential is varied [1, 2]. This is an example of a quantum phase transition [3] where quantum fluctuations and correlations rather than energy-entropy competition is the driving force and its theoretical understanding is quite challenging. The model usually considered for describing this phenomenon is the Bose-Hubbard model and the transition is interpreted as the superfluid-insulator transition that was studied in [4] with an application to $\mathrm{He}^{4}$ in porous media in mind. The possibility of applying this scheme to gases of alkali atoms in optical traps was first realized in [5]. The article [6] reviews these developments and many recent papers, e.g., 7, 8, 9, 10, 11, 12, 13, 14 are devoted to this topic. These papers contain also further references to earlier work along these lines.

The investigations of the phase transition in the BoseHubbard model are mostly based on variational or numerical methods and the signal of the phase transition is usually taken to be that an ansatz with a sharp particle number at each lattice site leads to a lower energy than a delocalized Bogoliubov state. On the other hand, there exists no rigorous proof, so far, that the true ground state of the model has off-diagonal long range order at one end of the parameter regime that disappears at the other end. In the present paper we study a slightly different model

\footnotetext{
* On leave from Department of Mathematics, University of Copen-
} hagen, Universitetsparken 5, DK-2100 Copenhagen, Denmark where just this phenomenon can be rigorously proved and which, at the same time, captures the salient features of the experimental situation.

The model is that of a hard core gas on a cubic lattice at half-filling (i.e., when the particle number is half the number of sites). The 'optical lattice' is modeled simply by a periodic, one-body potential $\lambda(-1)^{\mathbf{x}}$, where $(-1)^{\mathbf{x}}=+1$ on the A-sublattice and $(-1)^{\mathbf{x}}=-1$ on the B-sublattice. Thus, the Hamiltonian, expressed through bosonic creation and annihilation operators, equals

$$
\begin{aligned}
H= & -\frac{1}{2} \sum_{\langle\mathbf{x} \mathbf{y}\rangle}\left(a_{\mathbf{x}}^{\dagger} a_{\mathbf{y}}+a_{\mathbf{x}} a_{\mathbf{y}}^{\dagger}\right)+\lambda \sum_{\mathbf{x}}(-1)^{\mathbf{x}} a_{\mathbf{x}}^{\dagger} a_{\mathbf{x}} \\
& +U \sum_{\mathbf{x}} a_{\mathbf{x}}^{\dagger} a_{\mathbf{x}}\left(a_{\mathbf{x}}^{\dagger} a_{\mathbf{x}}-1\right) .
\end{aligned}
$$

The sites $\mathbf{x}$ are in a $d$-dimensional hypercubic lattice, and $\langle\mathbf{x y}\rangle$ stands for pairs of nearest neighbors. The case considered in this paper is the hard-core interaction $U=$ $\infty$. Apart from the periodic potential, this is also the Bose-Hubbard model with infinite on-site repulsion.

Note that in our model the troughs of the optical potential correspond to the B-sublattice where the periodic potential is negative, and the crests correspond to the Asublattice. Often in the Bose-Hubbard model the whole lattice itself is used to approximate the troughs alone. Roughly speaking, half-filling in our model corresponds to filling factor 1 in the Bose-Hubbard approximation.

As is well known, the model (1) with $U=\infty$ can also be viewed as the XY model of a spin $1 / 2$ system [15]. The periodic potential then corresponds to a staggered magnetic field. This will be explained in the next section.

We are able to prove the following facts rigorously for 3 or more dimensions. The $\lambda$-T-phase diagram at halffilling (e.g., mean density $\varrho=\frac{1}{2}$ ) is shown schematically in Figure 1 
1. If both $\lambda$ and the temperature $T$ are small, then there is Bose-Einstein condensation (BEC). In this parameter regime the one-body density matrix has exactly one large eigenvalue (in the thermodynamic limit), and the corresponding condensate wave function is $\varphi(\mathbf{x})=$ const.

2. If either $T$ or $\lambda$ is big enough, then the correlation function (the one-body density matrix) decays exponentially, and hence there is no BEC. In particular, this applies to the ground state $(T=0)$ for $\lambda$ big enough.

3. The Mott insulator phase is characterized by a gap, i.e., a jump in the chemical potential (at zero temperature). We are able to prove this, at half-filling, for big enough $\lambda$. More precisely, there is a cusp in the dependence of the ground state energy on the number of particles; adding or removing one particle costs a non-zero amount of energy. We also show that there is no such gap whenever there is BEC.

$\lambda$

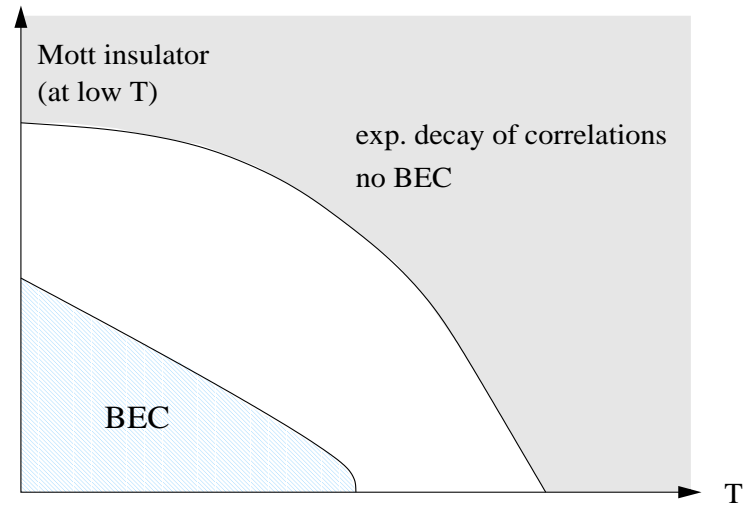

FIG. 1: Schematic phase diagram at half-filling

4. The interparticle interaction is essential for items 2 and 3. Non-interacting bosons always display BEC for low, but positive $T$ (which depends on $\lambda$, of course).

5. For all $T \geq 0$ and all $\lambda>0$ the diagonal part of the one-body density matrix $\left\langle a_{\mathbf{x}}^{\dagger} a_{\mathbf{x}}\right\rangle$ is not constant. Its value on the A-sublattice is constant, but strictly less than its constant value on the Bsublattice (for a finite system with periodic boundary conditions) and this discrepancy survives in the thermodynamic limit. In contrast, in the regime mentioned in item 1 the off-diagonal long-range order is constant, i.e., $\left\langle a_{\mathbf{x}}^{\dagger} a_{\mathbf{y}}\right\rangle \approx$ const. for large $|\mathbf{x}-\mathbf{y}|$.

We give explicit expressions for the curves, sketched in Figure 1 defining the regimes for which the above statements are proved here (see Eqs. (31) and (13) below.)
We are not able to make a rigorous statement about the intermediate regime, but we believe that there is only a critical line separating the BEC and the Mott insulator phases.

We focus here on lattice dimensions $d \geq 3$ but, using the technique employed in [16], an extension to the ground state in two dimensions is possible. Other possible extensions are mentioned in the next section.

\section{DETAILED DESCRIPTION OF THE MODEL}

We write the Hamiltonian (11) of the lattice gas with $U=\infty$ in terms of the creation and annihilation operators, $a_{\mathbf{x}}^{\dagger}$ and $a_{\mathbf{x}}$, for particles at lattice site $\mathbf{x} \in \Lambda$, with $\Lambda$ a finite hypercubic lattice with $L^{d}$ sites, $L$ being an even integer. We impose periodic boundary conditions. Because of the hard-core condition, there is at most one particle at each site, and thus the creation and annihilation operators can be represented as $2 \times 2$ matrices with

$$
a_{\mathrm{x}}^{\dagger} \leftrightarrow\left(\begin{array}{cc}
0 & 1 \\
0 & 0
\end{array}\right), \quad a_{\mathbf{x}} \leftrightarrow\left(\begin{array}{cc}
0 & 0 \\
1 & 0
\end{array}\right), \quad a_{\mathbf{x}}^{\dagger} a_{\mathbf{x}} \leftrightarrow\left(\begin{array}{ll}
1 & 0 \\
0 & 0
\end{array}\right),
$$

for each $\mathbf{x} \in \Lambda$. The correspondence with the spin matrices

$$
S^{1}=\frac{1}{2}\left(\begin{array}{ll}
0 & 1 \\
1 & 0
\end{array}\right), S^{2}=\frac{1}{2}\left(\begin{array}{cc}
0 & -\mathrm{i} \\
\mathrm{i} & 0
\end{array}\right), S^{3}=\frac{1}{2}\left(\begin{array}{cc}
1 & 0 \\
0 & -1
\end{array}\right)
$$

is

$$
a_{\mathbf{x}}^{\dagger}=S_{\mathbf{x}}^{1}+\mathrm{i} S_{\mathbf{x}}^{2} \equiv S_{\mathbf{x}}^{+}, \quad a_{\mathbf{x}}=S_{\mathbf{x}}^{1}-\mathrm{i} S_{\mathbf{x}}^{2} \equiv S_{\mathbf{x}}^{-},
$$

and hence $a_{\mathbf{x}}^{\dagger} a_{\mathbf{x}}=S_{\mathbf{x}}^{3}+\frac{1}{2}$. Adding a convenient constant to make the periodic potential positive, the Hamiltonian (11) for $U=\infty$ is thus equivalent to

$$
\begin{aligned}
H & =-\frac{1}{2} \sum_{\langle\mathbf{x y}\rangle}\left(S_{\mathbf{x}}^{+} S_{\mathbf{y}}^{-}+S_{\mathbf{x}}^{-} S_{\mathbf{y}}^{+}\right)+\lambda \sum_{\mathbf{x}}\left[\frac{1}{2}+(-1)^{\mathbf{x}} S_{\mathbf{x}}^{3}\right] \\
& =-\sum_{\langle\mathbf{x y}\rangle}\left(S_{\mathbf{x}}^{1} S_{\mathbf{y}}^{1}+S_{\mathbf{x}}^{2} S_{\mathbf{y}}^{2}\right)+\lambda \sum_{\mathbf{x}}\left[\frac{1}{2}+(-1)^{\mathbf{x}} S_{\mathbf{x}}^{3}\right] .
\end{aligned}
$$

As explained in the introduction, $(-1)^{\mathbf{x}}= \pm 1$ on alternating sites. Without loss of generality we may assume $\lambda \geq 0$. Note that the subtraction of the 'diagonal' terms in the kinetic energy has the effect of a chemical potential and as a consequence the unique ground state of (2) has particle number $N=\frac{1}{2}|\Lambda|$. We postpone the proof of this assertion to Appendix A

The presence or absence of Bose-Einstein condensation is expressed through the reduced one-particle density matrix

$$
\gamma(\mathbf{x}, \mathbf{y})=\left\langle a_{\mathbf{x}}^{\dagger} a_{\mathbf{y}}\right\rangle
$$

where $\langle\cdot\rangle$ denotes the expectation value in the thermal equilibrium state or the ground state considered. BEC occurs (by definition) if the $L^{d} \times L^{d}$ matrix $\gamma(\mathbf{x}, \mathbf{y})$ has 
an eigenvalue of order $N$ in the thermodynamic limit $\Lambda \rightarrow \infty, N \rightarrow \infty$, with $\varrho=N /|\Lambda|$ fixed.

We shall prove that for $\varrho=1 / 2$ and $d \geq 3$ the thermal equilibrium state of (2) shows Bose-Einstein condensation for small $\lambda$ and low temperature $T$, while for large $\lambda$ or $T$ the condensation disappears. For $d=2$ this is true only in the ground state. Here, $\varrho$ stands for the average density, since we are using the grand-canonical ensemble where the particle number is not fixed. Note that we are not dealing here with a dilute system and the condensation is always depleted, even in the ground state.

We remark that the Hamiltonian (2) is invariant under the following two unitary transformations, which will be used throughout the paper. 1) Uniform rotation around the $S^{3}$-axis, in particular the map $S_{\mathbf{x}}^{1} \rightarrow-S_{\mathbf{x}}^{1}$ and $S_{\mathrm{x}}^{2} \rightarrow-S_{\mathrm{x}}^{2}$ at all sites. The corresponding conserved generator is the total particle number. 2) The particle-hole symmetry, which corresponds to: $S_{\mathbf{x}}^{1} \leftrightarrow S_{\mathbf{x}}^{2}, S_{\mathbf{x}}^{3} \rightarrow-S_{\mathbf{x}}^{3}$ at all sites, followed by a unit-vector translation in any of the lattice directions. For the latter symmetry the half-filling is essential.

Our analysis of the system proceeds via the following steps. In the next Section III we prove infrared bounds on the two-point function $\left\langle\widetilde{S}_{\mathbf{p}}^{1} \widetilde{S}_{-\mathbf{p}}^{1}+\widetilde{S}_{\mathbf{p}}^{2} \widetilde{S}_{-\mathbf{p}}^{2}\right\rangle$ in momentumspace. The essential ingredient here is reflection positivity of the Hamiltonian (2) and the closely related property of Gaussian domination [17]. The bound obtained depends on $\lambda$ and the temperature. A sum rule fixes the sum over $\mathbf{p}$ of the two-point function and the infrared bound leads to the conclusion that for small $\lambda$ and low temperatures the contribution from $\mathbf{p}=\mathbf{0}$ remains non-vanishing in the thermodynamic limit. This proves BEC.

Reflection positivity is essential for our proof of BEC and forces the periodic potential to have period 2. Other generalizations can be accommodated, however, such as a more general lattice in which we add hopping to next nearest neighbors [18] or the addition of nearest neighbor interparticle repulsion [17]. For simplicity we concentrate here on the simple cubic lattice with on-site repulsion only.

In Section IV we show that the existence of BEC implies the absence of an energy gap for adding or removing a particle, and that the energy viewed as a function of the density has a unique tangent at $\varrho=\frac{1}{2}$, i.e., the chemical potential is continuous.

The absence of BEC for large $\lambda$ or high temperatures $T$ is proved in Section $\nabla$ The technique applied here is a path space representation of the two-point function that follows from the Trotter product formula. This representation allows us to derive exponential decay of the twopoint function, provided $\lambda$ or $T$ are sufficiently large, and hence absence of long range order. The magnitude of $\lambda$ or $T$ enters through the suppression in the path space integral of long contours connecting a pair of lattice points if these parameters are large. The same representation is used for proving the existence of a gap, as explained in item 3 in the introduction. This method is quite robust and easily extends to a periodicity of the optical lattice potential different from 2, for instance.

In Section VI we show that the particle density (or 3 -component of the spin) oscillates with the period of the staggered field if $\lambda \neq 0$, in contrast to the condensate wave function which is independent of $\lambda$ and $\mathbf{x}$. For this effect the interaction is essential, as remarked in Section VII Without the interaction there is always BEC (for low $T$ ) and the condensate wave function is never constant (for $\lambda \neq 0$ ).

In Appendix $\AA$ we shall prove that the ground state of (2) has total spin 0, which in the lattice gas language means that the lowest energy of (2) is obtained when the particle number is $|\Lambda| / 2$. The essential ingredient in our proof is again reflection positivity of the Hamiltonian (2). We also show that the canonical partition function is maximal at half-filling.

\section{PROOF OF BEC FOR SMALL $\lambda$ AND $T$}

In this section we are going to show the occurrence of BEC for small $\lambda$ and low enough temperature. The main result is the following.

THEOREM 1 (Existence of BEC). Let $E_{\mathbf{p}}=$ $\sum_{i=1}^{d}\left(1-\cos \left(p_{i}\right)\right)$ (where $p_{i}$ denotes the components of p) and

$$
c_{d}=\frac{1}{(2 \pi)^{d}} \int_{[-\pi, \pi]^{d}} d \mathbf{p} \frac{1}{E_{\mathbf{p}}} .
$$

In the thermodynamic limit,

$$
\begin{aligned}
& \lim _{\Lambda \rightarrow \infty} \frac{1}{|\Lambda|^{2}} \sum_{\mathbf{x}, \mathbf{y} \in \Lambda} \gamma(\mathbf{x}, \mathbf{y}) \\
& \geq \frac{1}{2}-\frac{1}{2}\left(\frac{1}{2}\left[d(d+1)+4 \lambda^{2}\right]^{1 / 2} c_{d}\right)^{1 / 2}-\frac{1}{\beta} c_{d}
\end{aligned}
$$

with $\beta=1 /\left(k_{\mathrm{B}} T\right)$ the inverse temperature. Moreover, if $\varphi(\mathbf{x})$ denotes the (normalized) eigenfunction corresponding to the largest eigenvalue of $\gamma(\mathbf{x}, \mathbf{y})$, then $\lim _{\Lambda \rightarrow \infty}|\Lambda|^{-1}\left|\sum_{\mathbf{x}} \varphi(\mathbf{x})\right|^{2}=1$, implying that the condensate wave function is constant in the thermodynamic limit.

Note that $c_{d}$ is finite for $d \geq 3$. Since the largest eigenvalue of $\gamma(\mathbf{x}, \mathbf{y})$ exceeds $|\Lambda|^{-1} \sum_{\mathbf{x}, \mathbf{y}} \gamma(\mathbf{x}, \mathbf{y})$, BEC is proved if the right side of the expression (3) is positive. This is in particular the case, for large enough $\beta$, as long as

$$
\lambda^{2}<\frac{1}{c_{d}^{2}}-\frac{d(d+1)}{4} .
$$

In $d=3, c_{3} \approx 0.505$ [17, and hence there is BEC for $\lambda \lesssim 0.960$. In [17] it was also shown that $d c_{d}$ is monotone decreasing in $d$, which implies a similar result for all $d>$ 3 . 
The main tool in our proof of Theorem 1 is an infrared bound as in 17]. The statement is as follows. For $A$ and $B$ bounded linear operators, denote by

$$
(A, B)=\int_{0}^{1} \operatorname{Tr}\left(A e^{-s \beta H} B e^{-(1-s) \beta H}\right) d s / \operatorname{Tr} e^{-\beta H}
$$

the Duhamel two-point function. For $\mathbf{p} \in \Lambda^{*}$ (the dual lattice), $\mathbf{p} \neq \mathbf{0}$ and $\widetilde{S}_{\mathbf{p}}^{1}=|\Lambda|^{-1 / 2} \sum_{\mathbf{x}} S_{\mathbf{x}}^{1} \exp (\mathbf{i p} \cdot \mathbf{x})$, we claim that

$$
\left(\widetilde{S}_{\mathbf{p}}^{1}, \widetilde{S}_{-\mathbf{p}}^{1}\right) \leq \frac{1}{2 \beta E_{\mathbf{p}}}
$$

The same is true with $\widetilde{S}_{\mathbf{p}}^{1}$ replaced by $\widetilde{S}_{\mathbf{p}}^{2}$. This inequality will allow us to prove the bound (3) above. Moreover, we will infer from (4) the fact that there is only one large eigenvalue (of order $|\Lambda|$ ) of the one-particle density matrix, and the corresponding eigenfunction is constant (in the thermodynamic limit).

We start by proving (4). The main ingredient is Gaussian domination. More precisely, let $h$ be any real-valued function on $\Lambda$, and

$$
Z(h)=\operatorname{Tr} \exp [-\beta K(h)],
$$

where $K(h)$ is the modified Hamiltonian

$$
\begin{aligned}
K(h)= & \sum_{\langle\mathbf{x y}\rangle}\left(\frac{1}{2}\left(S_{\mathbf{x}}^{1}-S_{\mathbf{y}}^{1}-h_{\mathbf{x}}+h_{\mathbf{y}}\right)^{2}-S_{\mathbf{x}}^{2} S_{\mathbf{y}}^{2}\right) \\
& +\lambda \sum_{\mathbf{x}}(-1)^{\mathbf{x}} S_{\mathbf{x}}^{3} .
\end{aligned}
$$

Note that for $h=0$ this operator agrees with $H$ up to a constant.

Lemma 1. For all real-valued functions $h$,

$$
Z(h) \leq Z(0) .
$$

Proof. We perform a unitary transformation that takes $S^{2} \mapsto-S^{2}$ and $S^{3} \mapsto-S^{3}$ on the B-sublattice. Since the trace does not change under unitary transformations, we have $Z(h)=\operatorname{Tr} \exp [-\beta \widehat{K}(h)]$ with

$$
\widehat{K}(h)=\sum_{\langle\mathbf{x y}\rangle}\left(\frac{1}{2}\left(S_{\mathbf{x}}^{1}-S_{\mathbf{y}}^{1}-h_{\mathbf{x}}+h_{\mathbf{y}}\right)^{2}+S_{\mathbf{x}}^{2} S_{\mathbf{y}}^{2}\right)+\lambda \sum_{\mathbf{x}} S_{\mathbf{x}}^{3} .
$$

Compared to $K(h)$, the sign in front of the $S_{\mathbf{x}}^{2} S_{\mathbf{y}}^{2}$ term has changed, and the $(-1)^{\mathbf{x}}$ has vanished. The operator $\widehat{K}(h)$ thus obtained is translation invariant. Since $S^{1}$ and $S^{3}$ are real self-adjoint matrices, and $S^{2}$ is imaginary and self-adjoint, we meet exactly the conditions for applying the result in 17, Lemma 6.1 and proof of Theorem 4.2] to prove the lemma.

The infrared bound (4) follows from this lemma by using the negativity of the second derivative,

$$
\left.\frac{d^{2}}{d \varepsilon^{2}} Z(\varepsilon h)\right|_{\varepsilon=0} \leq 0
$$

By performing the derivative, we obtain

$$
\left(A^{\dagger}, A\right) \leq \frac{1}{\beta} \sum_{\langle\mathbf{x y}\rangle}\left|h_{\mathbf{x}}-h_{\mathbf{y}}\right|^{2},
$$

where $A=\sum_{\langle\mathbf{x y}\rangle}\left(S_{\mathbf{x}}^{1}-S_{\mathbf{y}}^{1}\right)\left(h_{\mathbf{x}}-h_{\mathbf{y}}\right)$. We proved this inequality only for real-valued $h$, in which case $A=A^{\dagger}$, but it automatically extends in a standard way [17] to complex-valued $h$. In this case note that the adjoint of $A$ agrees with its complex conjugate. Now, choosing $h_{\mathbf{x}}=\exp (\mathbf{i p} \cdot \mathbf{x})$, we obtain (4). By invariance of the Hamiltonian under rotations around the $S^{3}$-axis, the statement is also true with $\widetilde{S}_{\mathbf{p}}^{1}$ replaced by $\widetilde{S}_{\mathbf{p}}^{2}$.

We now want to use [17, Theorem 3.1] to relate the Duhamel two-point function to the ordinary thermal twopoint function. For that purpose, we have to evaluate the double commutators

$$
\begin{aligned}
& {\left[\widetilde{S}_{\mathbf{p}}^{1},\left[H, \widetilde{S}_{-\mathbf{p}}^{1}\right]\right]+\left[\widetilde{S}_{\mathbf{p}}^{2},\left[H, \widetilde{S}_{-\mathbf{p}}^{2}\right]\right]} \\
& =-\frac{2}{|\Lambda|}\left(H-\frac{1}{2} \lambda|\Lambda|+2 \sum_{\langle\mathbf{x y}\rangle} S_{\mathbf{x}}^{3} S_{\mathbf{y}}^{3} \cos \mathbf{p} \cdot(\mathbf{x}-\mathbf{y})\right) .
\end{aligned}
$$

Let $C_{\mathbf{p}}$ denote the expectation value of this last expression,

$$
C_{\mathbf{p}}=\left\langle\left[\widetilde{S}_{\mathbf{p}}^{1},\left[H, \widetilde{S}_{-\mathbf{p}}^{1}\right]\right]+\left[\widetilde{S}_{\mathbf{p}}^{2},\left[H, \widetilde{S}_{-\mathbf{p}}^{2}\right]\right]\right\rangle \geq 0 .
$$

The positivity of $C_{\mathbf{p}}$ can be seen from an eigenfunctionexpansion of the trace. From [17, Corollary 3.2 and Theorem 3.2] and (4) we infer that

$$
\left\langle\widetilde{S}_{\mathbf{p}}^{1} \widetilde{S}_{-\mathbf{p}}^{1}+\widetilde{S}_{\mathbf{p}}^{2} \widetilde{S}_{-\mathbf{p}}^{2}\right\rangle \leq \frac{1}{2} \sqrt{\frac{C_{\mathbf{p}}}{E_{\mathbf{p}}}} \operatorname{coth} \sqrt{\beta^{2} C_{\mathbf{p}} E_{\mathbf{p}} / 4} .
$$

Using coth $x \leq 1+1 / x$ and Schwarz's inequality, we obtain for the sum over all $\mathbf{p} \neq \mathbf{0}$,

$$
\begin{aligned}
& \sum_{\mathbf{p} \neq \mathbf{0}}\left\langle\widetilde{S}_{\mathbf{p}}^{1} \widetilde{S}_{-\mathbf{p}}^{1}+\widetilde{S}_{\mathbf{p}}^{2} \widetilde{S}_{-\mathbf{p}}^{2}\right\rangle \\
& \leq \frac{1}{\beta} \sum_{\mathbf{p} \neq \mathbf{0}} \frac{1}{E_{\mathbf{p}}}+\frac{1}{2}\left(\sum_{\mathbf{p} \neq \mathbf{0}} \frac{1}{E_{\mathbf{p}}}\right)^{1 / 2}\left(\sum_{\mathbf{p} \neq \mathbf{0}} C_{\mathbf{p}}\right)^{1 / 2} .
\end{aligned}
$$

We have $\sum_{\mathbf{p} \in \Lambda^{*}} C_{\mathbf{p}}=-2\langle H\rangle+\lambda|\Lambda|$, which can be bounded from above using the following lemma. Its proof follows exactly the same lines as 17, Theorem C.1].

Lemma 2. The lowest eigenvalue of

$$
-\frac{1}{2} S_{\mathbf{x}}^{1} \sum_{i=1}^{2 d} S_{\mathbf{y}_{i}}^{1}-\frac{1}{2} S_{\mathbf{x}}^{2} \sum_{i=1}^{2 d} S_{\mathbf{y}_{i}}^{2}+\lambda S_{\mathbf{x}}^{3}
$$

is given by $-\frac{1}{4}\left[d(d+1)+4 \lambda^{2}\right]^{1 / 2}$.

Since the Hamiltonian can be written as a sum of terms like (6), with $\mathbf{y}_{i}$ the nearest neighbors of $\mathbf{x}$, we get from this lemma the lower bound

$$
H \geq-\frac{|\Lambda|}{4}\left[d(d+1)+4 \lambda^{2}\right]^{1 / 2}+\frac{1}{2} \lambda|\Lambda| .
$$


With the aid of the sum rule

$$
\sum_{\mathbf{p} \in \Lambda^{*}}\left\langle\widetilde{S}_{\mathbf{p}}^{1} \widetilde{S}_{-\mathbf{p}}^{1}+\widetilde{S}_{\mathbf{p}}^{2} \widetilde{S}_{-\mathbf{p}}^{2}\right\rangle=\frac{|\Lambda|}{2}
$$

(which follows from $\left(S^{1}\right)^{2}=\left(S^{2}\right)^{2}=1 / 4$ ), we obtain a lower bound, in the thermodynamic limit,

$$
\begin{aligned}
& \lim _{\Lambda \rightarrow \infty} \frac{1}{|\Lambda|}\left\langle\widetilde{S}_{\mathbf{0}}^{1} \widetilde{S}_{\mathbf{0}}^{1}+\widetilde{S}_{\mathbf{0}}^{2} \widetilde{S}_{\mathbf{0}}^{2}\right\rangle \\
& \geq \frac{1}{2}-\frac{1}{2}\left(\frac{1}{2}\left[d(d+1)+4 \lambda^{2}\right]^{1 / 2} c_{d}\right)^{1 / 2}-\frac{1}{\beta} c_{d} .
\end{aligned}
$$

The connection with Bose-Einstein condensation is as follows. Since $H$ is real, also $\gamma(\mathbf{x}, \mathbf{y})$ is real and we have

$$
\gamma(\mathbf{x}, \mathbf{y})=\left\langle S_{\mathbf{x}}^{+} S_{\mathbf{y}}^{-}\right\rangle=\left\langle S_{\mathbf{x}}^{1} S_{\mathbf{y}}^{1}+S_{\mathbf{x}}^{2} S_{\mathbf{y}}^{2}\right\rangle
$$

Hence, if $\varphi_{0}=|\Lambda|^{-1 / 2}$ denotes the constant function,

$$
\left\langle\varphi_{0}|\gamma| \varphi_{0}\right\rangle=\left\langle\widetilde{S}_{\mathbf{0}}^{1} \widetilde{S}_{\mathbf{0}}^{1}+\widetilde{S}_{\mathbf{0}}^{2} \widetilde{S}_{\mathbf{0}}^{2}\right\rangle
$$

and thus the bound (7) implies (3). In addition we claim that the infrared bounds imply

$$
\langle\varphi|\gamma| \varphi\rangle \leq \text { const. }|\Lambda|^{2 / d}
$$

for any normalized $\varphi$ that is orthogonal to $\varphi_{0}$, with a constant that is independent of $\varphi$. To see this, consider the positive definite matrix $\left\langle\widetilde{S}_{\mathbf{p}}^{+} \widetilde{S}_{-\mathbf{q}}^{-}\right\rangle$, with $\mathbf{p} \neq \mathbf{0}, \mathbf{q} \neq$ 0. The infrared bound (5) implies that the diagonal of this matrix is bounded by $\left\langle\widetilde{S}_{\mathbf{p}}^{+} \widetilde{S}_{-\mathbf{p}}^{-}\right\rangle \leq$const. $|\mathbf{p}|^{-2} \leq$ const. $|\Lambda|^{2 / d}$ for $\mathbf{p} \neq \mathbf{0}$. Moreover, the matrix is almost diagonal in the sense that $\left\langle\widetilde{S}_{\mathbf{p}}^{+} \widetilde{S}_{-\mathbf{q}}^{-}\right\rangle \neq 0$ only if $q_{i}=p_{i}$ or $q_{i}=p_{i} \pm \pi$ (by invariance under translation by two lattice sites). The largest eigenvalue of such a matrix is bounded above by $2^{d}$ times the maximum on the diagonal, namely $2^{d}$ const. $|\Lambda|^{2 / d} \ll|\Lambda|$. This proves our claim.

We conclude that $\gamma(\mathbf{x}, \mathbf{y})$ has exactly one large eigenvalue, with corresponding eigenfunction equal to $\varphi_{0}$ as $|\Lambda| \rightarrow \infty$. I.e., the condensate wave function is constant. This is in contrast to the particle density, which shows the staggering of the periodic potential. We show this in Section VI below. It also contrasts with the situation for zero interparticle interaction, as discussed in Section DII

\section{NO CUSP, NO GAP}

The system is in a Mott insulator state at zero temperature if a finite change in the chemical potential is required to change the particle number in the ground state. We refer to this as a gap in the chemical potential. More precisely, if $E_{k}$ denotes the lowest energy of (2) restricted to the sector of $\frac{1}{2}|\Lambda|+k$ particles (which corresponds to $S_{\text {tot }}^{3} \equiv \sum_{\mathbf{x}} S_{\mathbf{x}}^{3}=k$ in the spin language), a gap means that, for all $k$,

$$
E_{-k}+E_{k}-2 E_{0} \geq c|k|
$$

for some $c>0$ independent of $\Lambda$ and $k$. Note that particle-hole symmetry implies that $E_{-k}+E_{k}-2 E_{0}=$ $2\left(E_{|k|}-E_{0}\right)$.

In the next section we prove (8) for sufficiently large $\lambda$. In this section we will show that whenever there is BEC then (8) fails. In fact, we will prove that

$$
E_{k}-E_{0} \leq \frac{c_{k}}{|\Lambda|}
$$

for some $k$-dependent $c_{k}>0$, which is independent of $\Lambda$, i.e., that (8) does not hold for finite $k$.

This, however, does not rule out the possibility that the macroscopic system still acts as an insulator. To show that this is, indeed, also not the case, we prove that (8) fails for macroscopic $k$ as well. More precisely, we will show that the thermodynamic limit of the ground state energy per site, $e_{\infty}(\varrho)=\lim _{\Lambda \rightarrow \infty} E_{k} /|\Lambda|$, where $k=\left(\varrho-\frac{1}{2}\right)|\Lambda|$, satisfies a bound

$$
0 \leq e_{\infty}(\varrho)-e_{\infty}\left(\frac{1}{2}\right) \leq \text { const. }\left(\varrho-\frac{1}{2}\right)^{2}
$$

for $\varrho$ close to $1 / 2$, i.e., that there is no macroscopic cusp in the energy at half-filling.

We will first prove (10). With $|0\rangle$ being the ground state of $H$, and with $\mathbf{y}$ some point in the lattice, consider the states

$$
\left|\psi_{\mathbf{y}}\right\rangle=e^{\mathrm{i} \varepsilon S_{\mathrm{tot}}^{2}}\left(S_{\mathbf{y}}^{1}+\frac{1}{2}\right)|0\rangle .
$$

The motivation is the following: we take the ground state and first project onto a given direction of $S^{1}$ on some site $\mathbf{y}$. If there is long-range order, this should imply that essentially all the spins point in this direction now. Then we rotate slightly around the $S^{2}$-axis. The particle number should then go up by $\varepsilon|\Lambda|$, but the energy only by $\varepsilon^{2}|\Lambda|$.

The norm of $\left|\psi_{\mathbf{y}}\right\rangle$ is given by

$$
\left\langle\psi_{\mathbf{y}} \mid \psi_{\mathbf{y}}\right\rangle=\left\langle 0\left|S_{\mathbf{y}}^{1}+\frac{1}{2}\right| 0\right\rangle=\frac{1}{2},
$$

where we used the symmetry $S^{1,2} \rightarrow-S^{1,2}$. We want to find an upper bound to the average energy of these states, more precisely,

$$
\Delta E \equiv \frac{2}{|\Lambda|} \sum_{\mathbf{y}}\left\langle\psi_{\mathbf{y}}\left|H-E_{0}\right| \psi_{\mathbf{y}}\right\rangle .
$$

Here $E_{0}$ denotes the ground state energy of $H$, which is obtained at half-filling (see Appendix A). We claim that the inequality

$$
e^{-\mathrm{i} \varepsilon S_{\text {tot }}^{2}} H e^{\mathrm{i} \varepsilon S_{\text {tot }}^{2}} \leq H+\mathrm{i} \varepsilon\left[H, S_{\text {tot }}^{2}\right]+\text { const. } \varepsilon^{2}|\Lambda|
$$

holds for some constant depending only on $d$ and $\lambda$. To see this, consider, for self-adjoint operators $A$ and $C$,

$$
F_{A}(\varepsilon)=e^{\mathrm{i} \varepsilon C} A e^{-\mathrm{i} \varepsilon C} .
$$

By Taylor's formula

$$
F_{A}(\varepsilon) \leq F_{A}(0)+\varepsilon F_{A}^{\prime}(0)+\frac{1}{2} \varepsilon^{2} \sup _{0 \leq \eta \leq \varepsilon}\left\|F_{A}^{\prime \prime}(\eta)\right\| .
$$


Note that the last norm is actually independent of $\eta$, since $e^{-\mathrm{i} \varepsilon C}$ is unitary, and is given by the norm of the double commutator $[C,[C, A]]$. After evaluating the double commutator for the case in question, a simple bound gives (11).

Consider now the first term on the right side of (11). We obtain

$$
\begin{aligned}
& \sum_{\mathbf{y}}\left\langle 0\left|\left(S_{\mathbf{y}}^{1}+\frac{1}{2}\right)\left(H-E_{0}\right)\left(S_{\mathbf{y}}^{1}+\frac{1}{2}\right)\right| 0\right\rangle \\
& =\frac{1}{2} \sum_{\mathbf{y}}\left\langle 0\left|\left[S_{\mathbf{y}}^{1},\left[H, S_{\mathbf{y}}^{1}\right]\right]\right| 0\right\rangle \\
& =\frac{1}{2}\left\langle 0\left|\sum_{\langle\mathbf{x y}\rangle} 2 S_{\mathbf{x}}^{2} S_{\mathbf{y}}^{2}-\lambda \sum_{\mathbf{y}}(-1)^{\mathbf{y}} S_{\mathbf{y}}^{3}\right| 0\right\rangle \\
& =-\frac{1}{2}\left(E_{0}-\frac{1}{2} \lambda|\Lambda|\right),
\end{aligned}
$$

where we used rotational symmetry in the last step. The second term,

$$
\sum_{\mathbf{y}}\left\langle 0\left|\left(S_{\mathbf{y}}^{1}+\frac{1}{2}\right)\left[H, S_{\mathrm{tot}}^{2}\right]\left(S_{\mathbf{y}}^{1}+\frac{1}{2}\right)\right| 0\right\rangle
$$

is zero by symmetry, as can be seen in the following way. The diagonal terms are zero by the symmetry $S_{\mathbf{x}}^{1,2} \rightarrow$ $-S_{\mathbf{x}}^{1,2}$ at all sites. The off-diagonal terms are

$$
\left\langle 0\left|S_{\text {tot }}^{1}\left[H, S_{\text {tot }}^{2}\right]+\left[H, S_{\text {tot }}^{2}\right] S_{\text {tot }}^{1}\right| 0\right\rangle,
$$

which is zero by the symmetry $S_{\mathbf{x}}^{1,3} \rightarrow-S_{\mathbf{x}}^{1,3}$ at all sites, followed by a unit-vector translation in any of the lattice directions. We therefore get that

$$
\Delta E \leq \text { const. } \varepsilon^{2}|\Lambda|+\frac{\left|E_{0}\right|+\frac{1}{2} \lambda|\Lambda|}{|\Lambda|} .
$$

It remains to evaluate the average particle number of the states considered. Using that $S_{\text {tot }}^{3}|0\rangle=0$, we obtain

$$
\begin{aligned}
& \frac{2}{|\Lambda|} \sum_{\mathbf{y}}\left\langle 0\left|\left(S_{\mathbf{y}}^{1}+\frac{1}{2}\right) e^{-\mathrm{i} \varepsilon S_{\mathrm{tot}}^{2}} S_{\mathrm{tot}}^{3} e^{\mathrm{i} \varepsilon S_{\mathrm{tot}}^{2}}\left(S_{\mathbf{y}}^{1}+\frac{1}{2}\right)\right| 0\right\rangle \\
& =\frac{2}{|\Lambda|} \sin \varepsilon\left\langle 0\left|\left(S_{\mathrm{tot}}^{1}\right)^{2}\right| 0\right\rangle,
\end{aligned}
$$

which is of the order $\varepsilon|\Lambda|$ if there is BEC. Choosing $\varepsilon$ proportional to the chemical potential $\mu$, we obtain as an upper bound for the ground state energy of $H-\mu S_{\text {tot }}^{3}$

$$
E_{0}\left(1-|\Lambda|^{-1}\right)+\frac{1}{2} \lambda-c \mu^{2}|\Lambda|
$$

for small $\mu$, with $c>0$ in the case of BEC. By taking a Legendre transform, we arrive at (10).

To prove (9) we use as a trial state $\left(S_{\text {tot }}^{+}\right)^{k}|0\rangle$, with $k \geq 1$. Using the particle-hole symmetry of $H$ as well as the fact that we are considering the ground state, we get the bound

$$
E_{k} \leq E_{0}+\frac{1}{2} \frac{\left\langle\left[\left(S_{\mathrm{tot}}^{-}\right)^{k},\left[H,\left(S_{\mathrm{tot}}^{+}\right)^{k}\right]\right]\right\rangle}{\left\langle\left(S_{\mathrm{tot}}^{-}\right)^{k}\left(S_{\mathrm{tot}}^{+}\right)^{k}\right\rangle} .
$$

Since there is BEC, $\left\langle\left(S_{\text {tot }}^{-}\right)^{k}\left(S_{\text {tot }}^{+}\right)^{k}\right\rangle \geq c_{k}^{\prime}|\Lambda|^{2 k}$ for some $c_{k}^{\prime}>0$ modulo lower order terms as $|\Lambda| \rightarrow \infty$. All we have to show is that $\left[\left(S_{\text {tot }}^{-}\right)^{k},\left[H,\left(S_{\text {tot }}^{+}\right)^{k}\right]\right] \leq c_{k}^{\prime \prime}|\Lambda|^{2 k-1}$ for some constant $c_{k}^{\prime \prime}$. This is clear, however, since altogether there are $|\Lambda|^{2 k+1}$ factors, and the 2 commutators reduce the power by two. Hence we obtain (9).

\section{GAP FOR LARGE $\lambda$, AND ABSENCE OF BEC FOR LARGE $\lambda$ OR HIGH TEMPERATURE}

We shall now present explicit bounds for a region of values of $(\lambda, T)$ for which BEC is absent. This region includes:

(i) all $\lambda \geq 0$ at $k_{\mathrm{B}} T>d /(2 \ln 2)$,

(ii) all $T \geq 0$ at $\lambda \geq 0$ such that $\lambda+|e(\lambda)|>d$.

The absolute (i.e., without specifying $N$ ) ground state energy per site for a finite $\Lambda$, which is always obtained at half-filling, is denoted by $e(\lambda)$. Note that $e(\lambda)<0$.

In this regime, the particles are localized in the sense that the transition amplitudes decay exponentially. Short excursions occur locally in "space-time", however a long distance transition requires a linked chain, or percolation, of such local events and the amplitude for that decays exponentially, as in sub-critical percolation models. One may discern here two distinct mechanisms contributing to the localization: at high $\lambda$ localization is caused by the confinement due to the staggered structure of the potential, whereas at high temperatures it is a combined effect of the exclusion (no more than one particle at a lattice site) with the reduced amplitude for coordinated moves by neighboring particle. The above picture is made precise in a representation of the matrix elements of $e^{-\beta H}$ which in effect involves an "imaginary time".

A more inclusive statement of the condition under which our results hold is

$$
\beta>\frac{-1}{\lambda-f} \ln \left(1-\frac{\lambda-f}{d}\right),
$$

where $f \equiv f(\beta, \lambda)=-(\beta|\Lambda|)^{-1} \ln \operatorname{Tr} e^{-\beta H}$ is the free energy per site, which satisfies

$$
-f(\beta, \lambda) \geq \max \left\{|e(\lambda)|, \beta^{-1} \ln 2-\frac{1}{2} \lambda\right\} .
$$

Under condition (13), we define $\nu>0$ by

$$
e^{-\nu} \equiv \frac{d}{\lambda-f}\left(1-e^{-\beta(\lambda-f)}\right)<1 .
$$

THEOREM 2 (Mott insulator phase). Throughout the regime where (13) holds the thermal average two-point function decays exponentially. More specifically, for any $\xi<\nu$

$$
\gamma(\mathbf{x}, \mathbf{y})=\frac{\operatorname{Tr} a_{\mathbf{x}}^{\dagger} a_{\mathbf{y}} e^{-\beta H}}{\operatorname{Tr} e^{-\beta H}} \leq C_{\xi} e^{-\xi|\mathbf{x}-\mathbf{y}|}
$$


with $C_{\xi}=\left[1-e^{\xi-\nu}\right]^{-1}$. Similar decay also holds in the finite volume ground state (corresponding to the limit $\beta \rightarrow \infty$ in (14)).

Moreover, the ground state energy $E_{k}$ for particle number $\frac{1}{2}|\Lambda|+k$ satisfies

$$
E_{k}+E_{-k}-2 E_{0}=2\left(E_{k}-E_{0}\right) \geq c|k|
$$

with $c=2(\lambda+|e(\lambda)|-d)$, which is strictly positive for large $\lambda$ (independently of the volume $|\Lambda|$ ).

Eq. (15) is, in fact, the consequence of a more explicit result: If $\mathcal{P}_{k}$ denotes the projection onto the subspace of fixed particle number $N=\frac{1}{2}|\Lambda|+k$, then

$$
\frac{\operatorname{Tr} \mathcal{P}_{k} e^{-\beta H}}{\operatorname{Tr} \mathcal{P}_{0} e^{-\beta H}} \leq 2 e^{-\alpha \beta|k|}\left[\frac{e^{2}}{1-B(\alpha)} \frac{|\Lambda|}{|k|}\right]^{|k|},
$$

for any $\alpha>0$ for which

$$
B(\alpha) \equiv d \int_{0}^{\beta} e^{-\left(\lambda+\left|f_{0}(\beta, \lambda)\right|-\alpha\right) t} d t<1,
$$

with $f_{0}(\beta, \lambda)$ denoting the free energy for the system with fixed particle number $N=|\Lambda| / 2$. Eq. (15) is derived by considering the leading terms in (16) in the limit $\beta \rightarrow \infty$ at fixed $\Lambda$. In the thermodynamic limit Eq. (15) means that, in contrast to Eqs. (9) and (10), the energy per site, $e_{\infty}(\varrho)$, has a cusp at $\varrho=1 / 2$, and hence (by Legendre transform) the half filled state $\varrho=1 / 2$ corresponds to a whole interval of values for the chemical potential.

For non-zero temperature the energy dependence on $k$ may show some rounding due to thermal excitations, however there is a cusp in the energy per site, when this function is viewed on a scale in which $|k| /|\Lambda| \geq e^{-r \beta c}$, with some $r<1$.

Theorem 2 is derived using a representation for the matrix elements of the relevant operators in the basis that diagonalizes $\left\{S_{\mathbf{x}}^{3}\right\}$. An important fact here is that the matrix elements of $e^{-\beta H}$ are positive in this basis. The natural expansion for these matrix elements, e.g., via the Lie-Trotter formula, yields a functional-integral representation in terms of integrals over "space-time" configurations, which are represented below by $\omega$. The resulting functional integral is not only positive, but also reflection positive, and we make use of that fact. However, reflection positivity is not essential for the qualitative picture, as even without it we would obtain similar results with only slightly weaker bounds - with $f$ replaced by 0 .

The measure space over which the integration takes place is a Cartesian product of the set of initial spin configurations times the space of configurations of "rungs", linking pairs of neighboring "space time" sites. A rung is parametrized by a pair $\{\mathbf{x}, \mathbf{y}\}$ of neighboring lattice sites and $t \in[0, \beta]$. For the matrix elements of $e^{-\beta H}$ between states which correspond to a pair of specified spin configurations, one naturally finds an integral over configurations of arbitrary number of rungs, over which we integrate with an "ideal gas"-like measure, in which $n$ tuples are summed and integrated over with the weights $\frac{z^{n}}{n !} d t_{1} \cdots d t_{n}$. Each rung represents a transformation of the spin configuration affected by a specific term in the Hamiltonian, and the fugacity-like parameter $z$ is the corresponding amplitude, which in the case of the Hamiltonian considered here is $z=1 / 2$.

It is particularly convenient to express the spin, or particle, configuration in terms of the time-lines of the 'quasi-particles' which are defined through the occupation numbers $n_{\mathbf{x}}=\frac{1}{2}+(-1)^{\mathbf{x}} S_{\mathbf{x}}^{3}$. There are no quasiparticles in the configuration that minimizes the potential energy, i.e, if there are $\frac{1}{2}|\Lambda|$ particles that sit on the B-sublattice. The presence of a quasi-particle means either the presence of a particle $\left(S^{3}=+1 / 2\right)$, if the site is even (A-sublattice), or the absence of one $\left(S^{3}=-1 / 2\right)$, if the site is odd (B-sublattice). It is easy to check that in this representation the operators $a_{\mathbf{x}}^{\dagger}$ and $a_{\mathbf{x}}$ act as insertion of a source and, correspondingly, sink of excess spin (relative to the potential-minimizing configuration), although the direction in which the excess spin propagates changes with the parity of $\mathbf{x}$. Namely, creation of a particle on an A site (or annihilation on a B site) results in a quasiparticle running 'upward in time', whereas a quasiparticle running 'downward in time' originates from annihilation of a particle on an A site (or creation on a B site).

Proceeding along the above lines, as explained in greater detail in [19], one obtains

$$
\operatorname{Tr} \mathcal{P}_{k} e^{-\beta H}=\int v_{1 / 2}(d \omega) e^{-\lambda|\omega|} I[\nu(\omega)=k],
$$

where $\omega$ represents a configuration of a family of disjoint oriented loops in $\Lambda \times[0, \beta]$, defined with periodic boundary conditions in 'time' $([0, \beta])$, whose orientation alternates with $\mathbf{x}$, being 'up' along A sites and 'down' along B sites. For each configuration, $|\omega|$ is the total 'vertical' length of the time lines in $\omega$, and $\nu(\omega)$ is the total winding number in the periodic 'time' direction. The indicator function $I[\nu(\omega)=k]$ is 1 if the loop configuration $\omega$ has total winding number $k$, and 0 otherwise. The winding number can also be computed by adding the spin orientations of the sites occupied by quasi-particles, along any 'constant time' cut through the diagram. The measure $v_{z}(d \omega)$ corresponds to integration, with weights $z d t$, over the times at which the jumps to neighboring lattice sites occur, and summation over the possible numbers of such jumps. In effect, as mentioned above, the integral is over an 'ideal gas'-like distribution of the horizontal rungs in the diagram depicted in Figure 2 with the fugacity parameter taking here the value $z=1 / 2$. That value is dictated by the Hamiltonian, where one finds $1 / 2$ in front of the 'hopping term' $S_{\mathbf{x}}^{+} S_{\mathbf{y}}^{-}$. For later use, we find it convenient to consider the measures $v_{z}(d \omega)$ for general $z>0$, not only $z=1 / 2$.

Likewise, for $\mathbf{x} \neq \mathbf{y}$,

$$
\begin{aligned}
& \operatorname{Tr} a_{\mathbf{x}}^{\dagger} a_{\mathbf{y}} \mathcal{P}_{k} e^{-\beta H} \\
& =\int_{\partial \omega=\delta_{(\mathbf{x}, 0)}-\delta_{(\mathbf{y}, 0)}} v_{1 / 2}(d \omega) e^{-\lambda|\omega|} I[\nu(\omega)=k]
\end{aligned}
$$




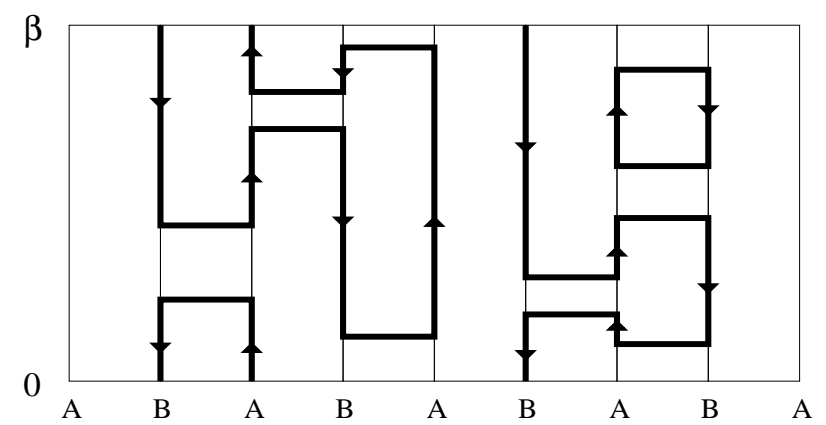

FIG. 2: Loop gas describing paths of quasi-particles for particle number $N=|\Lambda| / 2-1$. A line on an A site means presence of a particle, while on a $\mathrm{B}$ site it means absence. The horizontal rungs correspond to hopping of a particle.

where $\partial \omega$ is the set of sources of $\omega$. More precisely, the configurations that contribute to the last integral have exactly one curve starting at $\mathbf{x}$ and ending at $\mathbf{y}$, both at time 0 , for which we shall use the symbol $\gamma$, and otherwise only closed loops, of the kind which appear in the corresponding trace without the source/sink operators.

Denoting by $\mathcal{A}^{\mathrm{o}}$ the set of configurations $\omega$ that consist only of loops, i.e., closed curves, and by $\mathcal{A}^{(\mathbf{x}, \mathbf{y})}$ the set of configurations containing one curve connecting $\mathbf{x}$ with $\mathbf{y}$ (at time 0) and otherwise only closed curves, the above representation yields for the thermal average unconstrained by the particle number

$$
\left\langle S_{\mathbf{x}}^{+} S_{\mathbf{y}}^{-}\right\rangle=\frac{\int_{\mathcal{A}^{(\mathbf{x}, \mathbf{y})}} v_{1 / 2}(d \omega) e^{-\lambda|\omega|}}{\int_{\mathcal{A}^{\mathrm{o}}} v_{1 / 2}(d \omega) e^{-\lambda|\omega|}} .
$$

Now let $\mathcal{B}^{(\mathbf{x}, \mathbf{y})} \subset \mathcal{A}^{(\mathbf{x}, \mathbf{y})}$ denote the set of $\omega$ 's which consist of exactly one curve connecting $\mathbf{x}$ and $\mathbf{y}$, no other curves. For a collection of disjoint curves $\left\{\gamma_{j}\right\}$, let $\mathcal{A}_{\left\{\gamma_{j}\right\}}^{\mathrm{o}} \subset \mathcal{A}^{\mathrm{o}}$ denote the set of $\omega$ 's that avoid the collection $\left\{\gamma_{j}\right\}$ or, in other words, that are consistent with it in the sense that insertion of the curves into $\omega$ would still give an admissible configuration of non-intersecting curves. The measures $v_{z}(d \omega)$ obviously have the product structure

$$
\begin{aligned}
& \int_{\mathcal{A}^{(\mathbf{x}, \mathbf{y})}} v_{z}(d \omega) e^{-\lambda|\omega|} \\
& =\int_{\mathcal{B}^{(\mathbf{x}, \mathbf{y})}} v_{z}(d \gamma) e^{-\lambda|\gamma|} \int_{\mathcal{A}_{\gamma}^{\circ}} v_{z}(d \omega) e^{-\lambda|\omega|},
\end{aligned}
$$

where we identified $\omega$ with $\gamma$ in the first integral on the right. The following is a convenient bound on the last factor.

Lemma 3 (Contour bound). For any family of disjoint curves $\left\{\gamma_{j}\right\}$,

$$
\int_{\mathcal{A}_{\left\{\gamma_{j}\right\}}^{\mathrm{o}}} v_{z}(d \omega) e^{-\lambda|\omega|} \leq e^{\sum_{j}\left|\gamma_{j}\right| f} \int_{\mathcal{A}^{\circ}} v_{z}(d \omega) e^{-\lambda|\omega|},
$$

where $f$ is the free energy per site. Furthermore, a similar bound holds for the integrals further restricted by the condition $I[\nu(\omega)=0]$ and $f$ replaced by $f_{0}$ (which equals $\left.-(\beta|\Lambda|)^{-1} \ln \operatorname{Tr} \mathcal{P}_{0} e^{-\beta H}\right)$.

The proof, which is presented in Appendix B uses the chessboard inequality, which is a consequence of the reflection positivity of the functional integral. As was mentioned already, our main qualitative conclusions do not require this result, and would follow already from the trivial bound in which $f$ and $f_{0}$, which satisfy $f<f_{0}<0$, are replaced by 0 .

Lemma 3 implies that

$$
\left\langle S_{\mathbf{x}}^{+} S_{\mathbf{y}}^{-}\right\rangle \leq \int_{\mathcal{B}(\mathbf{x}, \mathbf{y})} v_{1 / 2}(d \gamma) e^{-|\gamma|(\lambda-f)} .
$$

To evaluate such expressions, it is useful to consider the quantity

$$
\chi(z, \lambda)=\sup _{\hat{x}, \hat{y}} \int_{\mathcal{B}^{(\hat{x}, \hat{y})}} v_{z}(d \gamma) e^{-\lambda|\gamma|},
$$

where $\hat{x}=\left(\mathbf{x}, t_{x}\right)$ and $\hat{y}=\left(\mathbf{y}, t_{y}\right)$ are arbitrary points in $\Lambda \times[0, \beta]$.

Lemma 4. If $2 z d \int_{0}^{\beta} e^{-\lambda t} d t<1$, then

$$
\chi(z, \lambda) \leq \frac{1}{1-2 z d \int_{0}^{\beta} e^{-\lambda t} d t} .
$$

Furthermore, for any $\{\hat{x}, \hat{y}\}$ and $\xi, \alpha>0$,

$$
\begin{aligned}
& \int_{\mathcal{B}^{(\hat{x}, \hat{y})}} v_{z}(d \gamma) e^{-\lambda|\gamma|} \leq e^{-\xi|\mathbf{x}-\mathbf{y}|} \chi\left(z e^{\xi}, \lambda\right), \\
& \int_{\mathcal{B}^{(\hat{x}, \hat{y})}} v_{z}(d \gamma) e^{-\lambda|\gamma|} I[|\gamma| \geq t] \leq e^{-\alpha t} \chi(z, \lambda-\alpha) .
\end{aligned}
$$

Proof. The first inequality here is a random walk bound, which is derived by the following 'renewal-type'argument: split the integral into a part that comes from curves that do not jump at all (which only occurs if $\mathbf{x}=\mathbf{y}$ ) and a part where $\gamma$ has at least one jump. The contribution from the path that does not jump is at most 1 . The first jump can be in $2 d$ possible directions, hence one gets

$$
\chi \leq 1+2 z d \int_{0}^{\beta} e^{-\lambda t} d t \chi
$$

Since in a finite volume $\chi<\infty$ a priori, this yields (22).

The rest follows by fairly direct arguments, noting that $v_{z e^{\xi}}(d \gamma)=v_{z}(d \gamma) e^{\xi \#(\gamma)}$, where $\#(\gamma)$ denotes the number of jumps of $\gamma$, which is greater or equal to $|\mathbf{x}-\mathbf{y}|$ in the case in question. The second inequality in (23) is obtained by estimating $I[|\gamma| \geq t] \leq e^{\alpha(|\gamma|-t)}$ for any positive $\alpha$.

We shall now use the above functional representation to derive Theorem 2 
By applying the bounds (21), (22) and (23) to the random walk representation (19), we see that under the condition stated in (13) the two-point function decays exponentially, as claimed in (14).

To prove (16) we start by noting that in case $N \neq|\Lambda| / 2$ the integral in (17) is over configurations with a nontrivial winding number, $\nu(\omega)=k$. Each such configuration includes a collection of 'non-contractible' loops $\left\{\gamma_{j}\right\}$ with non-zero winding numbers, $\nu_{j} \equiv \nu\left(\gamma_{j}\right) \neq 0$. The total length of the set $\left\{\gamma_{j}\right\}$ is at least $\beta|k|$. We shall bound the relative weight of such configurations by using the second bound in (23) and combining it with an argument whose purpose is to control the 'entropy' of such a collection of long loops.

Each non-contractible loop $\gamma_{j}$ can be labeled by a starting point $\mathbf{x}_{j} \in \Lambda$ where $\gamma_{j}$ crosses the 0 time line, and a winding number $\nu_{j}$. We shall actually overcount by summing over all possible $\mathbf{x}_{j}$ 's as starting points for the loops (with the only restriction that $\mathbf{x}_{j} \neq \mathbf{x}_{i}$ for $j \neq i$ ), and over all possible winding numbers $\nu_{j}$ with $\sum_{j}\left|\nu_{j}\right| \geq|k|$.

For a given collection $\left\{\gamma_{j}\right\}$ of non-contractible loops, we can bound the integral over the remaining loops by an integral over loops with zero total winding number that avoid the $\gamma_{i}$ 's. Hence, starting from (17), we get the bound

$$
\begin{aligned}
& \frac{\operatorname{Tr} \mathcal{P}_{k} e^{-\beta H}}{\operatorname{Tr} \mathcal{P}_{0} e^{-\beta H}} \leq \sum_{\left\{\mathbf{x}_{1}, \mathbf{x}_{2}, \ldots\right\} \subset \Lambda} \\
& \prod_{j} \int_{\mathcal{B}^{\left(\mathbf{x}_{j}, \mathbf{x}_{j}\right)}} v_{1 / 2}\left(d \gamma_{j}\right) e^{-\lambda\left|\gamma_{j}\right|} I\left[\nu\left(\gamma_{j}\right) \neq 0\right] \\
& \times I\left[\sum_{j}\left|\nu\left(\gamma_{j}\right)\right| \geq|k|\right] I_{\mathrm{ni}}\left[\left\{\gamma_{1}, \gamma_{2}, \ldots\right\}\right] \\
& \times \frac{\int_{\mathcal{A}_{\left\{\gamma_{j}\right\}}^{\circ}} v_{1 / 2}(d \omega) e^{-\lambda|\omega|} I[\nu(\omega)=0]}{\int_{\mathcal{A}^{\circ}} v_{1 / 2}(d \omega) e^{-\lambda|\omega|} I[\nu(\omega)=0]} .
\end{aligned}
$$

Here $I_{\text {ni }}$ denotes the indicator function for having only non-intersecting loops. Using the chessboard bound of Lemma 3 the last fraction can be bounded above by $\prod_{j} e^{f_{0}(\beta, \lambda)\left|\gamma_{j}\right|}$, with $f_{0}(\beta, \lambda)<0$ the free energy per site at $N=|\Lambda| / 2$. Applying the bounds of Lemma 4 to the integral over $n$ loops $\gamma_{i}$ with given absolute value of the winding number, $m_{i}=\left|\nu_{i}\right|$, we have, for any $\alpha>0$,

$$
\begin{aligned}
& \prod_{j=1}^{n} \int_{\mathcal{B}^{\left(\mathbf{x}_{j}, \mathbf{x}_{j}\right)}} v_{1 / 2}\left(d \gamma_{j}\right) e^{-\left(\lambda+\left|f_{0}\right|\right)\left|\gamma_{j}\right|} I\left[\left|\nu\left(\gamma_{j}\right)\right|=m_{j}\right] \\
& \leq \widehat{\chi}(\alpha)^{n} e^{-\alpha \beta \sum_{j} m_{j}} .
\end{aligned}
$$

Here $\widehat{\chi}(\alpha)=\chi\left(1 / 2, \lambda+\left|f_{0}(\beta, \lambda)\right|-\alpha\right)$, which is finite if $\alpha$ is not too large.

To complete the bound, we have to sum the right side of (24) over all the possible choices of the collection of the starting points of the winding loops $\left\{\mathbf{x}_{1}, \ldots, \mathbf{x}_{n}\right\}$, and over all possible winding numbers $\nu_{j}$ with $\left|\nu_{j}\right|>1$ and $\sum_{j}\left|\nu_{j}\right| \geq|k|$. To do so, we employ the following device.
Defining

$$
P(z)=1+\widehat{\chi}(\alpha) \sum_{i=1}^{|\Lambda| / 2}(z \delta)^{i},
$$

with $\delta=e^{-\alpha \beta}$, we see that the sum in question is given by the sum of all the coefficients of $z^{l}$ in $P(z)^{|\Lambda|}$ with powers $l \geq|k|$. Hence

$$
\frac{\operatorname{Tr} \mathcal{P}_{k} e^{-\beta H}}{\operatorname{Tr} \mathcal{P}_{0} e^{-\beta H}} \leq \frac{1}{2 \pi \mathrm{i}} \oint_{|z|=R} \frac{d z}{z^{|k|+1}} \frac{1}{1-z^{-1}} P(z)^{|\Lambda|},
$$

where $R$ can be any number greater than 1 . The contour integral serves as a filter, selecting for us the relevant coefficients of the polynomial $P(z)^{|\Lambda|}$. A simple bound shows that the above quantity is bounded from above by

$$
\inf _{1<R<1 / \delta} \frac{1}{1-R^{-1}} \frac{e^{|\Lambda| \widehat{\chi}(\alpha) \frac{R \delta}{1-R \delta}}}{R^{|k|}} .
$$

We now choose $R=k /(\delta \widehat{\chi}(\alpha)|\Lambda|)$, assuming that this quantity is greater or equal to 2 . Note that $\delta R \leq \frac{1}{2}$, since $|k| \leq \frac{1}{2}|\Lambda|$ and $\widehat{\chi}(\alpha) \geq 1$. Hence we obtain

$$
\frac{\operatorname{Tr} \mathcal{P}_{k} e^{-\beta H}}{\operatorname{Tr} \mathcal{P}_{0} e^{-\beta H}} \leq 2 e^{-\alpha \beta|k|}\left[e^{2} \widehat{\chi}(\alpha) \frac{|\Lambda|}{|k|}\right]^{|k|} .
$$

This inequality is also valid, however, if $|k| /(\delta \widehat{\chi}(\alpha)|\Lambda|)<$ 2 , since the resulting bound then exceeds 1 , which is greater than the left hand side (as shown in Appendix A). This proves the claim made in (16), which presents sufficient conditions for the existence of a cusp in the energy dependence on $N$, i.e., of a gap in the chemical potential.

\section{NON-CONSTANCY OF THE DENSITY}

In Section [II above we have demonstrated the existence of BEC for small $\lambda$ and $T$, and also that the condensate wave function is constant. Despite this fact the particle density has the periodicity of the external potential and is not constant for $\lambda \neq 0$. More precisely, the following result is proved below.

THEOREM 3 (Non-constancy of the density). Let $\varrho(\mathbf{x})=\gamma(\mathbf{x}, \mathbf{x})$ denote the particle density in the thermal equilibrium state at inverse temperature $\beta$. With $e(\lambda, \beta)=|\Lambda|^{-1}\langle H\rangle$ equal to the energy per site,

$$
\frac{1}{|\Lambda|}\left|\sum_{\mathbf{x} \in \Lambda}(-1)^{\mathbf{x}} \varrho(\mathbf{x})\right| \geq \frac{\lambda|e(0, \beta)|^{2}}{2 d^{2}(3 d+\lambda)} .
$$

We first prove this for the ground state. We will show that the ground state energy of $H$, denoted by $E(\lambda)$, satisfies

$$
E(\lambda) \leq E(0)+\frac{1}{2} \lambda|\Lambda|-c \lambda^{2}|\Lambda|,
$$


with $c=\frac{1}{2} e_{0}^{2} d^{-2} /(3 d+\lambda)$. (Here $e_{0}=E(0) /|\Lambda|$ denotes the ground state energy per site at $\lambda=0$.) Eq. (26) implies (25) by the following argument. Write $H=H_{0}+$ $\lambda W$, with the obvious notation for $H_{0}$ and $W$. Since $E(\lambda)$ is a concave function of $\lambda$, we have

$$
\langle W\rangle=E^{\prime}(\lambda) \leq \frac{E(\lambda)-E(0)}{\lambda} \leq \frac{1}{2}|\Lambda|-c \lambda|\Lambda| .
$$

On the other hand,

$$
\langle W\rangle=\sum_{\mathbf{x} \in \Lambda}\left[\frac{1}{2}+(-1)^{\mathbf{x}}\left\langle S_{\mathbf{x}}^{3}\right\rangle\right]=\frac{1}{2}|\Lambda|+\sum_{\mathbf{x} \in \Lambda}(-1)^{\mathbf{x}} \varrho(\mathbf{x}) .
$$

Combining the last two equations, we obtain

$$
\frac{1}{|\Lambda|}\left|\sum_{\mathbf{x} \in \Lambda}(-1)^{\mathbf{x}} \varrho(\mathbf{x})\right| \geq c \lambda,
$$

which proves our claim.

It remains to show (26). To do this, let the operator $C$ be given by

$$
C=\frac{1}{2} \sum_{\langle\mathbf{x y}\rangle}(-1)^{\mathbf{x}}\left(S_{\mathbf{x}}^{1} S_{\mathbf{y}}^{2}-S_{\mathbf{x}}^{2} S_{\mathbf{y}}^{1}\right) .
$$

As in the Hamiltonian, the sum is over all nearest neighbor pairs, each pair counted only once. This operator has the nice property that

$$
[C, W]=\mathrm{i} H_{0} .
$$

Proceeding as in (11)-(12), a simple bound of the relevant double commutators gives

$$
e^{\mathrm{i} \varepsilon C} W e^{-\mathrm{i} \varepsilon C} \leq W-\varepsilon H_{0}+\varepsilon^{2} \frac{d^{2}}{2}|\Lambda|
$$

as well as

$$
e^{\mathrm{i} \varepsilon C} H_{0} e^{-\mathrm{i} \varepsilon C} \leq H_{0}+\mathrm{i} \varepsilon\left[C, H_{0}\right]+\varepsilon^{2} \frac{3 d^{3}}{2}|\Lambda| .
$$

Note that the $\varepsilon^{2}$-terms are of order of the volume, due to the fact that both $C$ and $H$ contain only nearest neighbor terms. With $|0\rangle$ the ground state of $H_{0}$, we therefore have, using $\langle 0|W| 0\rangle=\frac{1}{2}|\Lambda|$ and $\left\langle 0\left|\left[C, H_{0}\right]\right| 0\right\rangle=0$,

$$
\begin{aligned}
E(\lambda) & \leq\left\langle 0\left|e^{\mathrm{i} \varepsilon C} H e^{-\mathrm{i} \varepsilon C}\right| 0\right\rangle \\
& \leq E(0)(1-\varepsilon \lambda)+\frac{1}{2} \lambda|\Lambda|+\frac{1}{2} \varepsilon^{2} d^{2}(3 d+\lambda)|\Lambda| .
\end{aligned}
$$

Now the optimal choice of $\varepsilon$ is $\varepsilon=2 c \lambda / e_{0}$, which finishes the proof of (26).

A similar argument works at positive temperature. It shows that the free energy depends non-trivially on $\lambda$, and by the same concavity argument as above this implies the non-constancy of the density, also at positive temperature, as claimed in (25).

\section{THE NON-INTERACTING GAS}

The interparticle interaction is essential for the existence of a Mott insulator phase for large $\lambda$. In case of absence of the hard-core interaction, there is BEC for any density and any $\lambda$ at low enough temperature (for $d \geq 3$ ). To see this, we have to calculate the spectrum of the one-particle Hamiltonian $-\frac{1}{2} \Delta+V(\mathbf{x})$, where $\Delta$ denotes the discrete Laplacian and $V(\mathbf{x})=\lambda(-1)^{\mathbf{x}}$. The spectrum can be easily obtained by noting that $V$ anticommutes with the off-diagonal part of the Laplacian, i.e., $\{V, \Delta+2 d\}=0$. Hence

$$
\left(-\frac{1}{2} \Delta-d+V(\mathbf{x})\right)^{2}=\left(-\frac{1}{2} \Delta-d\right)^{2}+\lambda^{2},
$$

so the spectrum is given by

$$
d \pm \sqrt{\left(\sum_{i} \cos p_{i}\right)^{2}+\lambda^{2}}
$$

where $\mathbf{p} \in \Lambda^{*}$. In particular, $E(\mathbf{p})-E(0) \sim \frac{1}{2} d\left(d^{2}+\right.$ $\left.\lambda^{2}\right)^{-1 / 2}|\mathbf{p}|^{2}$ for small $|\mathbf{p}|$, and hence there is BEC for low enough temperature. Note that the condensate wave function is of course not constant in this case, but rather given by the eigenfunction corresponding to the lowest eigenvalue of $-\frac{1}{2} \Delta+\lambda(-1)^{\mathbf{x}}$.

\section{CONCLUSION}

We have introduced a lattice model, which is similar to the usual Bose-Hubbard model and which describes the transition between Bose-Einstein condensation and a Mott insulator state as the strength $\lambda$ of the optical lattice potential is increased. While the model is not soluble in the usual sense, we can prove rigorously all the essential features that are observed experimentally. These include the existence of BEC for small $\lambda$ and its suppression for large $\lambda$, which is a localization phenomenon depending heavily on the fact that the Bose particles interact with each other. In the Mott insulator regime we prove the existence of a gap in the chemical potential, which does not exist in the BEC phase and for which the interaction is also essential. Bounds on the critical $\lambda$ as a function of temperature are included.

\section{Acknowledgments}

We are grateful to Letizzia Wastavino for help with Figures 1 and 2 The work was supported in part by the NSF grants PHY 9971149 (MA), PHY 0139984-A01 (EHL), and DMS-0111298 (JPS); by EU grant HPRNCT-2002-00277 (JPS and JY), by MaPhySto - A Network in Mathematical Physics and Stochastics funded by The Danish National Research Foundation (JPS), and by grants from the Danish research council (JPS). 


\section{APPENDIX A: HALF-FILLING AND REFLECTION POSITIVITY}

In this appendix we will show that $H$ has a unique ground state which has particle number $|\Lambda| / 2$. For $\lambda=0$, this was previously shown in [20]. We also establish the corresponding result at positive temperature, namely that the canonical partition function is maximal for particle number $N=|\Lambda| / 2$, although we do not prove that the maximum is obtained only at half-filling.

The operator $H$ commutes with $\sum_{\mathbf{x}} S_{\mathbf{x}}^{3}$. By a PerronFrobenius argument the ground state of $H$ restricted to the subspace with fixed value of $\sum_{\mathbf{x}} S_{\mathbf{x}}^{3}$ is unique. We claim that the absolute ground state of $H$ corresponds to the value $\sum_{\mathbf{x}} S_{\mathbf{x}}^{3}=0$. To prove this we shall use reflection positivity.

We divide the lattice in a left part and a right part $\Lambda=\Lambda_{L} \cup \Lambda_{R}$ of equal size. We shall identify the space $\mathcal{H}_{1}=\bigotimes_{\mathbf{x} \in \Lambda_{L}} \mathbb{C}^{2}$ with the space $\bigotimes_{\mathbf{x} \in \Lambda_{R}} \mathbb{C}^{2}$, by identifying factors reflected in the middle plane. We may therefore write the total Hilbert space as $\mathcal{H}=\mathcal{H}_{1} \otimes \mathcal{H}_{1}$. We may then write

$$
H=H_{L} \otimes I+I \otimes H_{R}-\frac{1}{2} \sum_{\langle\mathbf{x y}\rangle \in M}\left(S_{\mathbf{x}}^{+} S_{\mathbf{y}}^{-}+S_{\mathbf{x}}^{-} S_{\mathbf{y}}^{+}\right),
$$

where $H_{L}$ and $H_{R}$ act on $\mathcal{H}_{1}$ and $M$ denotes the set of bonds going from the left sublattice to the right sublattice (note that because of the periodic boundary condition these include the bonds that connect the right boundary with the left boundary). Note that $H_{L} \neq H_{R}$.

We now change $H$ to the unitarily equivalent operator $H^{\prime}$ for which at all sites on the right sublattice we change $S_{\mathbf{x}}^{ \pm} \rightarrow S_{\mathbf{x}}^{\mp}$ and $S_{\mathbf{x}}^{3} \rightarrow-S_{\mathbf{x}}^{3}$. We have

$$
H^{\prime}=H_{L} \otimes I+I \otimes H_{L}-\frac{1}{2} \sum_{\langle\mathbf{x y}\rangle \in M}\left(S_{\mathbf{x}}^{+} S_{\mathbf{y}}^{+}+S_{\mathbf{x}}^{-} S_{\mathbf{y}}^{-}\right) .
$$

The same unitary will change $\sum_{\mathbf{x}} S_{\mathbf{x}}^{3}$ to $S^{\prime}=S \otimes I-I \otimes S$, where $S=\sum_{\mathbf{x} \in \Lambda_{L}} S_{\mathbf{x}}^{3}$ acts on $\mathcal{H}_{1}$.

Let $|\psi\rangle \in \mathcal{H}_{1} \otimes \mathcal{H}_{1}$ be a normalized absolute ground state for $H^{\prime}$ with $S^{\prime}|\psi\rangle=m|\psi\rangle$. We want to show that $m=0$. We may write $|\psi\rangle=\sum_{n}\left|\psi_{n}\right\rangle$, where $S \otimes I\left|\psi_{n}\right\rangle=$ $n\left|\psi_{n}\right\rangle$ and $I \otimes S\left|\psi_{n}\right\rangle=(n-m)\left|\psi_{n}\right\rangle$.

We may consider any state $|\phi\rangle \in \mathcal{H}_{1} \otimes \mathcal{H}_{1}$ as an operator from the Hilbert space $\mathcal{H}_{1}$ to itself, in the following way. We introduce a basis $\mid X)$ in $\mathcal{H}_{1}$ indexed by subsets $X \subseteq \Lambda_{L}$, labeling the state with all spins up at the sites in $X$ and down elsewhere. We shall refer to this representation as the standard basis. Then $|\phi\rangle$ may be represented as a function associating a complex number $\phi(X, Y)$ to any pair of subsets $X, Y \subseteq \Lambda_{L}$, namely $\phi(X, Y)$ is given by the inner product of $\mid X) \otimes \mid Y$ ) with $|\phi\rangle$. Hence $|\phi\rangle$ may be identified with the operator $\widehat{\phi}$ defined by the matrix elements $(X|\widehat{\phi}| Y)=\phi(X, Y)$.

If $A$ is an operator on $\mathcal{H}_{1}$ then $\widehat{A \otimes I}|\psi\rangle=A \widehat{\psi}$ and $\widehat{I \otimes A \mid} \psi\rangle=\widehat{\psi} A^{T}$ where $A^{T}$ is the transposed operator represented by the matrix $A^{T}(X, Y)=A(Y, X)$ in the standard basis. (Note that transposition is not a canonical operation, but depends on the basis in which it is defined.)

The operator $S$ is represented by a real symmetric matrix in the standard basis. Thus in the above representation $\widehat{\psi}_{n}$ maps the subspace where $S=(n-m)$ to the subspace where $S=n$ and vanishes on the orthogonal complement. Hence we see that $\widehat{\psi}^{\dagger} \widehat{\psi}=\sum_{n} \widehat{\psi}_{n}^{\dagger} \widehat{\psi}_{n}$ and likewise $\widehat{\psi} \widehat{\psi}^{\dagger}=\sum_{n} \widehat{\psi}_{n} \widehat{\psi}_{n}^{\dagger}$. It follows from this that $S \widehat{\psi}^{\dagger} \widehat{\psi}=\widehat{\psi}^{\dagger} \widehat{\psi} S$ and $S \widehat{\psi} \widehat{\psi}^{\dagger}=\widehat{\psi} \widehat{\psi}^{\dagger} S$. Hence

$$
S\left(\widehat{\psi}^{\dagger} \widehat{\psi}\right)^{1 / 2}=\left(\widehat{\psi}^{\dagger} \widehat{\psi}\right)^{1 / 2} S, \quad S\left(\widehat{\psi} \widehat{\psi}^{\dagger}\right)^{1 / 2}=\left(\widehat{\psi} \widehat{\psi}^{\dagger}\right)^{1 / 2} S
$$

Let $\left|\psi_{1}\right\rangle,\left|\psi_{2}\right\rangle \in \mathcal{H}_{1} \otimes \mathcal{H}_{1}$ denote the states such that $\widehat{\psi}_{1}=\left(\widehat{\psi} \widehat{\psi}^{\dagger}\right)^{1 / 2}$ and $\widehat{\psi}_{2}=\left(\widehat{\psi}^{\dagger} \widehat{\psi}\right)^{1 / 2}$. Then $\left|\psi_{1}\right\rangle$ and $\left|\psi_{2}\right\rangle$ are normalized $\left(\right.$ since $\left\langle\psi_{1} \mid \psi_{1}\right\rangle=\operatorname{Tr}\left(\widehat{\psi}_{1}^{\dagger} \widehat{\psi}_{1}\right)=\operatorname{Tr}\left(\widehat{\psi} \widehat{\psi}^{\dagger}\right)=$ $\langle\psi \mid \psi\rangle$ ) and (A1) implies that $S^{\prime}\left|\psi_{1}\right\rangle=0$ and $S^{\prime}\left|\psi_{2}\right\rangle=0$.

We shall prove that

$$
\left\langle\psi\left|H^{\prime}\right| \psi\right\rangle \geq \frac{1}{2}\left\langle\psi_{1}\left|H^{\prime}\right| \psi_{1}\right\rangle+\frac{1}{2}\left\langle\psi_{2}\left|H^{\prime}\right| \psi_{2}\right\rangle
$$

Since $|\psi\rangle$ is an absolute ground state we see that $\left|\psi_{1}\right\rangle$ and $\left|\psi_{2}\right\rangle$ are also absolute ground states. Since they both have $S^{\prime}=0$ and the ground state with this property is unique we conclude that $\left|\psi_{1}\right\rangle=\left|\psi_{2}\right\rangle$, i.e., $\widehat{\psi}^{\dagger} \widehat{\psi}=\widehat{\psi} \widehat{\psi}^{\dagger}$. Then since we are using a representation in which the matrix for $S$ is real and symmetric we have

$$
\begin{aligned}
m & =\left\langle\psi\left|S^{\prime}\right| \psi\right\rangle=\langle\psi|(S \otimes I-I \otimes S)| \psi\rangle \\
& =\operatorname{Tr}\left(\widehat{\psi} \widehat{\psi}^{\dagger} S\right)-\operatorname{Tr}\left(\widehat{\psi}^{\dagger} \widehat{\psi} S\right)=0 .
\end{aligned}
$$

It remains to show the reflection positivity (A2). We may rewrite

$$
\begin{aligned}
& \left\langle\psi\left|H^{\prime}\right| \psi\right\rangle=\operatorname{Tr}\left(\widehat{\psi} \widehat{\psi}^{\dagger} H_{L}\right)+\operatorname{Tr}\left(\widehat{\psi}^{\dagger} \widehat{\psi} H_{L}\right) \\
& -\frac{1}{2} \sum_{\mathbf{x} \in M_{L}}\left(\operatorname{Tr}\left(\widehat{\psi}^{\dagger} S_{\mathbf{x}}^{+} \widehat{\psi} S_{\mathbf{x}}^{-}\right)+\operatorname{Tr}\left(\widehat{\psi}^{\dagger} S_{\mathbf{x}}^{-} \widehat{\psi} S_{\mathbf{x}}^{+}\right)\right) .
\end{aligned}
$$

Here $M_{L}$ denotes the set of sites in $\Lambda_{L}$ that connect to a bond in $M$, i.e., sites in $\Lambda_{L}$ that are nearest neighbor to a site in $\Lambda_{R}$. We have used that the operators $S_{\mathbf{x}}^{ \pm}$are represented by real matrices in the standard basis.

The inequality A2 now follows from the inequality

$$
\begin{aligned}
\operatorname{Tr}\left(\widehat{\psi}^{\dagger} A \widehat{\psi} A^{\dagger}\right) \leq & {\left[\operatorname{Tr}\left(\left(\widehat{\psi} \widehat{\psi}^{\dagger}\right)^{1 / 2} A\left(\widehat{\psi} \widehat{\psi}^{\dagger}\right)^{1 / 2} A^{\dagger}\right)\right]^{1 / 2} } \\
& \times\left[\operatorname{Tr}\left(\left(\widehat{\psi}^{\dagger} \widehat{\psi}\right)^{1 / 2} A\left(\widehat{\psi}^{\dagger} \widehat{\psi}\right)^{1 / 2} A^{\dagger}\right)\right]^{1 / 2}
\end{aligned}
$$

which holds for any operator $A$. This inequality is a simple application of the Cauchy-Schwarz inequality if one uses polar decomposition, i.e., the existence of a partial isometry $U$ such that

$$
\widehat{\psi}=U\left(\widehat{\psi}^{\dagger} \widehat{\psi}\right)^{1 / 2} \text { and }\left(\widehat{\psi} \widehat{\psi}^{\dagger}\right)^{1 / 2}=U\left(\widehat{\psi}^{\dagger} \widehat{\psi}\right)^{1 / 2} U^{\dagger}
$$


At positive temperature we may consider the partition function for $H$ restricted to the subspaces with fixed value of $\sum_{\mathbf{x}} S_{\mathbf{x}}^{3}$. We define

$$
Z(m)=\operatorname{Tr} \mathcal{P}_{m} \exp (-\beta H),
$$

where $\mathcal{P}_{m}$ is the projection onto the eigenspace of $\sum_{\mathbf{x}} S_{\mathbf{x}}^{3}$ corresponding to the eigenvalue $m$. We claim that the partition function is maximal at half-filling, i.e.,

$$
Z(m) \leq Z(0) .
$$

To prove this we shall again use reflection positivity.

We first note that the unitary change which mapped $H$ to $H^{\prime}$ will take $\mathcal{P}_{m}$ into the operator

$$
\mathcal{P}_{m}^{\prime}=\sum_{n} P_{n} \otimes P_{n-m}
$$

where $P_{n}$ is the projection operator in $\mathcal{H}_{1}$ projecting onto the eigenspace of $S$ with eigenvalue $n$. Observe that $P_{m}$ is a real matrix in the standard basis.

Using the Trotter product formula we may now write

$$
\begin{aligned}
& Z(m)=\lim _{k \rightarrow \infty} \operatorname{Tr} \mathcal{P}_{m}^{\prime} \times \\
& \quad\left(e^{-\frac{\beta}{k} H_{L} \otimes I} e^{-\frac{\beta}{k} I \otimes H_{L}} e^{\frac{\beta}{2 k} \sum_{M} S_{\mathbf{x}}^{+} S_{\mathbf{y}}^{+}} e^{\frac{\beta}{2 k} \sum_{M} S_{\mathbf{x}}^{-} S_{\mathbf{y}}^{-}}\right)^{k} .
\end{aligned}
$$

If we use that

$$
e^{\frac{\beta}{2 k} \sum_{\langle\mathbf{x} \mathbf{y}\rangle \in M} S_{\mathbf{x}}^{ \pm} S_{\mathbf{y}}^{ \pm}}=\prod_{\langle\mathbf{x y}\rangle \in M}\left(1+\frac{\beta}{2 k} S_{\mathbf{x}}^{ \pm} S_{\mathbf{y}}^{ \pm}\right)
$$

we see that the trace above may be written as sums of terms of the form $A_{n} A_{n-m}$, where

$$
A_{n}=\operatorname{Tr}_{\mathcal{H}_{1}}\left(P_{n} e^{-\beta H_{L} / k} T_{1} e^{-\beta H_{L} / k} T_{2} \cdots e^{-\beta H_{L} / k} T_{k}\right),
$$

and each of the operators $T_{1}, T_{2} \ldots$ is a monomial in the variables $(\beta / 2 k)^{1 / 2} S_{\mathbf{x}}^{ \pm}, \mathbf{x} \in M_{L}$.

Since $A_{n}$ is real for all $n$ we see that $A_{n} A_{n-m} \leq A_{n}^{2} / 2+$ $A_{n-m}^{2} / 2$. If we insert this above and simply undo the calculation we arrive at (A4).

\section{APPENDIX B: REFLECTION POSITIVITY CONTOUR BOUND}

In this appendix we derive Lemma 3 using reflection positivity arguments.

The measures $v_{z}(d \omega)$ are reflection positive in the following sense. Draw a hyperplane, either vertically (through bonds) or horizontally, that divides $\Gamma=\Lambda \times$ $[0, \beta]$ into two congruent parts $\Gamma_{L} \cup \Gamma_{R}$. For any configuration $\omega$, let $\bar{\omega}$ be its natural reflection through the hyperplane (reversing its direction), and for any function $h$ on the space of configurations let $\widetilde{h}(\omega)=h(\bar{\omega})$. Then, for any such complex valued function that depends only on the restriction of $\omega$ to $\Gamma_{L}$,

$$
\int_{\mathcal{A}^{\circ}} h(\omega) \widetilde{h}(\omega)^{*} e^{-\lambda|\omega|} v_{z}(d \omega) \geq 0 .
$$

This can be seen by noting that once the behavior of $\omega$ on the hyperplane is fixed, the distribution of the left and right sides (or top and bottom) are conditionally independent, and are mirror images of each other.

Reflection positivity leads to what is known as the chessboard inequality [21, 22]. In essence, it is a multiply reflected generalization of the Schwarz inequality, which allows us to obtain bounds on the expectation value of a product of local variables in terms of thermodynamic quantities.

The function whose average we need to estimate is $\chi_{D}(\omega)$ - the indicator function which is 1 if the curves in $\omega$ avoid a specified set $D \subset \Gamma$ and 0 otherwise. One may start by partitioning the imaginary time interval $[0, \beta]$, and correspondingly the "space-time" $\Gamma$, into equal short segments whose reflections tile $\Gamma$. For any subset $D \subset \Gamma$ that is a union of elements of the finite partition of $\Gamma$ the strategy, which is explained in detail in [21], yields

$$
\begin{aligned}
& \frac{\int_{\mathcal{A}^{\circ}} v_{z}(d \omega) \chi_{D}(\omega) e^{-\lambda|\omega|}}{\int_{\mathcal{A}^{\circ}} v_{z}(d \omega) e^{-\lambda|\omega|}} \\
& \quad \leq\left(\frac{\int_{\mathcal{A}^{\circ}} v_{z}(d \omega) \chi_{\Gamma}(\omega) e^{-\lambda|\omega|}}{\int_{\mathcal{A}^{\circ}} v_{z}(d \omega) e^{-\lambda|\omega|}}\right)^{|D| /|\Gamma|}=e^{f|D|},
\end{aligned}
$$

where $|D|$ is the total length of $D$. By refining the partition, and applying elementary continuity arguments (the dominated convergence theorem), we conclude that (B2) extends to all sets $D$ which are finite unions of closed intervals. This proves (20).

To prove the second statement in Lemma 3 we note that reflection positivity holds also for the restriction of the measure to $\omega$ 's with 0 winding number. I.e., for $h$ as in (B1),

$$
\int_{\mathcal{A}^{\circ}} h(\omega) \widetilde{h}(\omega)^{*} I[\nu(\omega)=0] e^{-\lambda|\omega|} v_{z}(d \omega) \geq 0 .
$$

If the hyperplane dividing $\Gamma$ into $\Gamma_{L} \cup \Gamma_{R}$ is horizontal, this is clear, since fixing $\omega$ on this hyperplane fixes the winding number. If it is vertical, however, we note that $I[\nu(\omega)=0]$ can be written as $\sum_{k} I_{k}(\omega) \widetilde{I}_{k}(\omega)$, where $k$ runs from 0 to $|\Lambda| / 2$, and $I_{k}$ is the indicator function for $\omega$ restricted to $\Gamma_{L}$ having winding number $k$. (Note that $\omega$ restricted to $\Gamma_{L}$ may have sources and sinks on the boundary, and when counting the winding number we also have to consider the curves resulting from these.) The second claim made in Lemma 3 follows by proceeding as in (B2), but with the added restriction to 0 winding number. 
[1] M. Greiner, O. Mandel, T. Esslinger, T.E. Hänsch, I. Bloch, Quantum phase transition from a superfluid to a Mott insulator in a gas of ultracold atoms, Nature 415, 39 (2002).

[2] M. Greiner, O. Mandel, T.E. Hänsch, I. Bloch, Collapse and revival of the matter wave field of a Bose-Einstein condensate, Nature 419, 51 (2002).

[3] S. Sachdev, Quantum Phase Transitions, Cambridge University Press, 1999.

[4] M.P.A. Fisher, P.B. Weichman, G. Grinstein, D.S. Fisher, Boson localization and the superfluid-insulator transition, Phys. Rev. B 40, 546-570 (1989).

[5] D. Jaksch, C. Bruder, J.I. Cirac, C.W. Gardiner, P. Zoller, Cold bosonic atoms in optical lattices, Phys. Rev. Lett. 81, 3108-3111 (1998).

[6] W. Zwerger, Mott-Hubbard transition of cold atoms in optical lattices, Journal of Optics B 5, 9-16 (2003).

[7] J.J. Garcia-Ripoll, J.I. Cirac, P. Zoller, C. Kollath, U. Schollwoeck, J. von Delft, Variational ansatz for the superfluid Mott-insulator transition in optical lattices, Arxiv: cond-mat/0306162 (2003).

[8] K. Ziegler, Phase Transition of a Bose Gas in an Optical Lattice, Laser Physics 13, 587-593 (2003).

[9] Z. Nazario, D.I. Santiago, Quantum States of Matter of Simple Bosonic Systems: BEC's, Superfluids and Quantum Solids, Arxiv: cond-mat/0308005 (2003).

[10] G.M. Genkin, Manipulating the superfluid - Mott insulator transition of a Bose-Einstein condensate in an amplitude-modulated optical lattice, Arxiv: cond-mat/ 0311589 (2003).

[11] K. Ziegler, Two-component Bose gas in an optical lattice at single-particle filling, Phys. Rev. A 68, 053602 (2003).

[12] D.B.M. Dickerscheid, D. van Oosten, P.J.H. Denteneer, H.T.C. Stoof, Ultracold atoms in optical lattices, Phys.
Rev. A 68, 043623 (2003).

[13] A.M. Rey, K. Burnett, R. Roth, M. Edwards, C.J. Williams, C.W. Clark, Bogoliubov approach to superfluidity of atoms in an optical lattice, J. Phys. B 36, 825-841 (2003).

[14] O. Morsch, E. Arimondo, Ultracold atoms and BoseEinstein condensates in optical lattices, Lecture Notes in Physics Vol. 602, Springer (2002).

[15] T. Matsubara, H. Matsuda, A lattice model of liquid helium, Progr. Theor. Phys. 16, 569-582 (1956).

[16] T. Kennedy, E.H. Lieb, S. Shastry, The XY Model has Long-Range Order for all Spins and all Dimensions Greater than One, Phys. Rev. Lett. 61, 2582-2584 (1988).

[17] F.J. Dyson, E.H. Lieb, B. Simon, Phase Transitions in Quantum Spin Systems with Isotropic and Nonisotropic Interactions, J. Stat. Phys. 18, 335-383 (1978).

[18] E.H. Lieb, P. Schupp, Ground State Properties of a Fully Frustrated Quantum Spin System, Phys. Rev. Lett. 83, 5362-5365 (1999). Singlets and reflection symmetric spin systems, Physica A 279, 378-385 (2000).

[19] M. Aizenman, B. Nachtergaele, Geometric Aspects of Quantum Spin States, Commun. Math. Phys. 164, 17-63 (1994).

[20] D.C. Mattis, Ground-State Symmetry in XY Model of Magnetism, Phys. Rev. Lett. 42, 1503-1505 (1979).

[21] J. Fröhlich, E.H. Lieb, Phase Transitions in Anisotropic Lattice Spin Systems, Commun. Math. Phys. 60, 233-267 (1978).

[22] J. Fröhlich, B. Simon, Pure States for General $\mathrm{P}(\Phi)_{2}$ Theories: Construction, Regularity and Variational Equality, Ann. Math. 105, 493 (1977). 